\title{
ABO-Incompatible Liver Transplantation in Acute Liver Failure: A Single Portuguese Center Study
}

\author{
M. Mendes, A.C. Ferreira, A. Ferreira, F. Remédio, I. Aires, A. Cordeiro, A. Mascarenhas, \\ A. Martins, P. Pereira, H. Gloria, R. Perdigoto, J. Veloso, P. Ferreira, J. Oliveira, M. Silva, E. Barroso, \\ and F. Nolasco
}

\begin{abstract}
Introduction. ABO-incompatible liver transplantation (ABOi LT) is considered to be a rescue option in emergency transplantation. Herein, we have reported our experience with ABOi LT including long-term survival and major complications in these situations.

Patient and Methods. ABOi LT was performed in cases of severe hepatic failure with imminent death. The standard immunosuppression consisted of basiliximab, corticosteroids, tacrolimus, and mycophenolate mofetil. Pretransplantation patients with anti-ABO titers above 16 underwent plasmapheresis. If the titer was above 128, intravenous immunoglobulin (IVIG) was added at the end of plasmapheresis. The therapeutic approach was based on the clinical situation, hepatic function, and titer evolution. A rapid increase in titer required five consecutive plasmapheresis sessions followed by administration of IVIG, and at the end of the fifth session, rituximab.
\end{abstract}

Results. From January 2009 to July 2012, 10 patients, including 4 men and 6 women of mean age 47.8 years (range, 29 to 64 years), underwent ABOi LT. At a mean follow-up of 19.6 months (range, 2 days to 39 months), 5 patients are alive including 4 with their original grafts. One patient was retransplanted at 9 months. Major complications were infections, which were responsible for 3 deaths due to multiorgan septic failure ( 2 during the first month); rejection episodes (4 biopsy-proven of humoral rejections in 3 patients and 1 cellular rejection) and biliary.

Conclusion. The use of ABOi LT as a life-saving procedure is justifiable in emergencies when no other donor is available. With careful recipient selection close monitoring of hemagglutinins and specific immunosuppression we have obtained acceptable outcomes.

\begin{abstract}
A CUTE hepatic failure (AHF) is associated with a high mortality rate. Emergency orthotopic liver transplantation (OLT) is the only durable life-saving treatment beyond the transitory supportive means of a critical care unit. ABO-incompatible liver transplantation (ABOi LT) which was reported in 1972 by Starz, ${ }^{1}$ has gained acceptance in Asia, ${ }^{2}$ North America, ${ }^{3}$ and Europe. ${ }^{4}$ Japan, the leading country performing ABOi LT to overcome the organs shortage, has investigated desensitization strategies. ${ }^{2,5}$ ABOi transplantation remains a high-risk procedure performed only in cases lacking an available/compatible organ donor $^{6}$ or as a rescue option in emergency situations with the unavailability of a compatible graft. ${ }^{7-11}$

Early results with adult ABOi LT were disappointing; patient and graft survivals were significantly worse than
\end{abstract}

adult compatible OLT. ${ }^{2,9,10}$ In 1988 , Demetris et al reported a $46 \%$ graft failure rate during the first 30 days after primary ABOi LT compared with $11 \%$ with ABO compatibility. ${ }^{12}$ In 1990, Guggenheim's group reported 30\% graft

From the Department of Nephrology (M.M., A.C.F., A.F., F.R., I.A., F.N.), Blood Center (A.C., A.M.), Department of Surgery (A.M., P.P., E.B.), Department of Gastroenterology (H.G., R.P., J.V.), and the Intensive Care Unit (P.F., J.O., M.S.), Centro Hospitalar de Lisboa Central, Hospital Curry Cabral, Lisbon, Portugal.

Address reprint requests to Marco Mendes, Department of Nephrology, Centro Hospitalar de Lisboa Central, Hospital Curry Cabral, Rua da Beneficência n8, 1069-166, Lisbon, Portugal. E-mail: marcomendes82@gmail.com 360 Park Avenue South, New York, NY 10010-1710 
function at 2 years after transplantation, whereas it was $76 \%$ for compatible ABO transplantations. ${ }^{7}$ The worse outcomes related to the potential for antibody-mediated rejection (AMR) associated with the presence of agglutinin antibodies. $^{12-15}$ Vascular thrombosis, acute liver necrosis, ${ }^{11-14}$ intrahepatic biliary complications, ${ }^{16-18}$ and sepsis caused by over-immunosuppression have been recognized to be causes of the poor outcomes. ${ }^{5,14}$ Recently, some authors have reported similar patient and graft survivals for ABOi LT compared with compatible OLT among all age groups. ${ }^{4,5,11,19}$ Two-year adult recipient survival after ABOi LT was reported to be nearly $70 \%$, whereas before 2002 , it was approximately $40 \% .^{5}$ Similar data have also been reported in a review of the Organ Procurement and Transplantation Network (OPTN) in 2009. ${ }^{3}$

In most clinical studies, two main strategies have been tested in combination to reduce antibody-mediated complications and prevent or ameliorate lesions caused by antigenantibody reactions. Recipient performed isoagglutinins can be reduced by plasmapheresis. ${ }^{6,13,14,19-21}$ or by immunoadsorption $^{22,23}$ with adjunctive treatment by splenectomy and/or rituximab. 4,5,14,14,24

In 2009, our insitution implemented the current protocol for ABOi LT for patients with AHF and multiorganic failure (MOF) who are judged to be unable to wait for a compatible graft.

\section{PATIENTS AND METHODS}

From January 2009 to July 2012, we performed 10 ABOi OLT: 4 men, 6 women, with mean age 47.8 years (range 29 to 64 years). During the same period, we performed a total of 300 liver transplantations. The demographic and clinical characteristics of the 10 ABOi LT patients are summarized in Table 1.

The indications for OLT in patient 1 were AHF secondary to imatinib treatment for chronic myeloid leukemia. Patients 2, 6, and 8 required retransplantation because of AHF. Patient 2 secondary to Budd-Chiari syndrome at 3 years after the first transplantation in the context of polycystic liver disease. Patient 6 secondary to failure of a first (at 3 months) and a second (at 9 days) transplantation in the context of chronic hepatic disease secondary to hepatitis B virus (HBV) and hepatitis $\mathrm{C}$ virus. In patient 8 , AHF was a result of alcoholic cirrhosis with a single hepatocellular carcinoma nodule already treated with quimioembolization. In the second case, failure of a first (at 3 months) and a second (at 9 days) transplantation in the context of chronic hepatic disease secondary to hepatitis $\mathrm{B}$ virus (HBV) and hepatitic $\mathrm{C}$ virus. In the third case, hepatocellular carcinoma (HCC) as a result of alcoholic cirrhosis and a single hepatocellular carcinoma nodule already treated with quimioembolization. Patients 3, 4, 7, and 10 developed AHF due to unknown causes. Patient 5 had AHF secondary to Amanita phalloides; patient 9 , secondary to autoimmune hepatitis.

Donor-to-recipient $\mathrm{ABO}$ matches were $\mathrm{AB}$ to $\mathrm{O}+,(\mathrm{n}=4 ; 40 \%)$ $\mathrm{A}+$ to $0+(\mathrm{n}=2 ; 20 \%), \mathrm{A}$ to $\mathrm{B}(\mathrm{n}=2 ; 20 \%)$ A to $0(\mathrm{n}=1 ; 10 \%)$ and $\mathrm{B}$ to $\mathrm{A}(\mathrm{n}=1 ; 10 \%)$.

The patients all displayed severe hepatic failure with an average model for end-stage liver disease (MELD) score of 37 (range, 19 to 53). They were listed for OLT on appeal, but progressive worsening of their clinical conditions associated with MOF and hemodynamic instability did not allow a delay in the timing of OLT. At the time of transplantation, $3(30 \%)$ received dialysis, $7(70 \%)$ patients at least one session of prometheus, $7(70 \%)$ were ventilated, and 8 $(80 \%)$ were under aminergic support.

\section{Immunosuppressive Protocol}

Our protocol was based on those reported in the literature for ABOi kidney transplantation and ABOi LT using living donors. The immunosuppressive regimen consisted of induction therapy with Basiliximab (Simulect, Novartis, Basel V; $20 \mathrm{mg} / \mathrm{iv}$ on days 1 and 4 ; methylprednisolone $(500 \mathrm{mg} / \mathrm{iv})$ on the first 3 days switched to oral prednisolone a $(1 \mathrm{mg} / \mathrm{kg} / \mathrm{d}$; maximum $60 \mathrm{mg} / \mathrm{d})$, tapered progressively); tacrolimus (Prograf, Astellas Pharma, Tokyo, Japan; of $0.05 \mathrm{mg} / \mathrm{kg}$ ) between days 1 and 4 progressively adjusted to achieve trough levels of 8 to $12 \mathrm{ng} / \mathrm{mL}$. Thereafter, the target was reduced to approximately $7 \mathrm{ng} / \mathrm{mL}$; and mycophenolate mofetil (CellCept, Roche Pharmaceuticals, Basel, Switzerland) administered at the daily dose of $1 \mathrm{~g}$, started on the third postoperative day. Tacrolimus and mycophenolate mofetil were first given intravenously, then switched to oral administration with the resumption of oral intake.

Related to the $\mathrm{ABO}$ incompatibility, the recipient's hemagglutinin titer, anti-A and anti-B, measurements were performed before OLT. Test erythrocytes suspended in serial doubling dilutions of recipient serum were centrifuged and evaluated for agglutination. The greatest unequivocally positive dilution was defined as the anti-A or -B antibody titer. ABO titers above 16 underwent plasmapheresis pretransplantation. The rationale for preoperative plasmapheresis to lower the anti-ABO titer below 1:16, and to keep

Table 1. Patient Characteristics

\begin{tabular}{|c|c|c|c|c|c|c|c|c|c|c|}
\hline $\begin{array}{l}\text { Patient } \\
\text { No. }\end{array}$ & Age & Gender & $\begin{array}{l}\text { Meld } \\
\text { Score }\end{array}$ & Original Disease & $\begin{array}{c}\text { ABO } \\
\text { Recipient }\end{array}$ & $\begin{array}{l}\text { ABO } \\
\text { Donor }\end{array}$ & PMT & LSE & Ventilated & $\begin{array}{c}\text { Aminergic } \\
\text { Support }\end{array}$ \\
\hline 1 & 29 & $\mathrm{~F}$ & 33 & Toxic Imatinib & $\mathrm{O}+$ & $A B$ & Yes & 3 & Yes & Yes \\
\hline 2 & 58 & $\mathrm{~F}$ & 39 & Retransplant Acute BCS & $\mathrm{O}+$ & $A B$ & No & 2 & Yes & Yes \\
\hline 3 & 52 & $\mathrm{~F}$ & 37 & AHF & $\mathrm{O}+$ & $A B+$ & Yes & 3 & Yes & Yes \\
\hline 4 & 32 & $\mathrm{~F}$ & 50 & $\mathrm{AHF}$ & $\mathrm{O}+$ & $\mathrm{A}+$ & Yes & 4 & Yes & Yes \\
\hline 5 & 43 & M & 53 & Toxic: Amanitas Phalloides & B & A & No & 3 & No & No \\
\hline 6 & 53 & M & 31 & Retransplant HCV, HBV & $\mathrm{O}$ & $A$ & Yes & 1 & Yes & Yes \\
\hline 7 & 38 & $\mathrm{~F}$ & 29 & $\mathrm{AHF}$ & $\mathrm{O}+$ & $A+$ & Yes & 2 & No & No \\
\hline 8 & 64 & M & 19 & Retransplant Alcoholic, HCC & $\mathrm{B}+$ & $\mathrm{A} 1$ & No & 4 & Yes & Yes \\
\hline 9 & 52 & $\mathrm{~F}$ & 35 & Autoimmune & $A+$ & $\mathrm{B}$ & Yes & 4 & No & Yes \\
\hline 10 & 58 & M & 44 & $\mathrm{AHF}$ & $\mathrm{O}+$ & $A B$ & Yes & 3 & Yes & Yes \\
\hline
\end{tabular}

Abbreviations: MELD, model for end-stage liver disease; PMT, prometheus; PSE, Portosystemic encephalopathy; BCS, Budd-chain syndrome; AHF, acute hepatic failure; HCV, hepatitis C virus; HBV, hepatitis B virus; HCC, hepatocellular carcinoma. 
it there after OLT was derived from the kidney transplant literature. Titers above 128 were treated with intravenous immunoglobulin IVIG $(2 \mathrm{~g} / \mathrm{kg})$ administered after the plasmapheresis. Hemagglutinin titers were performed daily posttransplantation for at least 21 days. The therapeutic approach was determined according to the clinical situation, the hepatic function, and the evolution of the ABO titers. Rapid increasing titers implied at least five consecutive plasmapheresis sessions followed by administration of IVIG (400 $\mathrm{mg} / \mathrm{kg} / \mathrm{d}$ ) after each one. At the end of the fifth session we infused rituximab $\left(375 \mathrm{~m} / \mathrm{m}^{2}\right)$.

Dual needle plasmapheresis procedures were performed to 1.0-1.5 exchange of the calculated plasma volumes using $100 \%$ fluid balances. The replacement fluids for plasma exchanges-fresh frozen plasma and 5\% human albumin-and their ratios were based on coagulation parameters.

The immunosuppressive regimen in each patient was heterogenerous (Table 2) because the protocol was adjusted to the clinical status of the patient, namely, hemodynamic instability or presence of infection.

\section{Histology}

Whenever clinical or biological signs suggested rejection, we performed liver biopsies. All liver biopsy specimens were subjected to paraffin block immunohitochemistry for complement derivative $4 \mathrm{~d}$ (C4d), hemagglutin deposition, and cytotoxic antibodies in sinusoids to investigate AMR. Acute cellular rejection (ACR) was defined according to Banff criteria.

\section{Infection Prophylaxis}

Antimicrobial prophylaxis consisted of piperacilin-tazobactam $(4.5 \mathrm{~g})$ every 8 hours for at least a week. For cytomegalovirus antiviral prophylaxis, the patients received valganciclovir $(900$ $\mathrm{mg} / \mathrm{d}$ ), for 3 months. Antifungal prophylaxis consisted of the administration of amphotericin B lipid complex $(1 \mathrm{mg} / \mathrm{kg} / \mathrm{d})$ for the first week, then $2.5 \mathrm{mg} / \mathrm{kg}$ twice a week for an additional 3 weeks. Pneumocystis Jiroveci prophylaxis consisted $(480 \mathrm{mg} / \mathrm{d})$ of trimethoprim/sulfamethoxazole $(480 \mathrm{mg} / \mathrm{d})$. Doses were adjusted to patient clinical status and laboratory results.

\section{RESULTS}

At a mean follow-up of 19.6 months (range, 2 days-39 months), 5 patients are alive, 4 of them carrying the original graft (Table 2). Patient survival was $50 \%$ and graft survival, $40 \%$.

\section{Complications}

Histology. Among the biopsies performed, four in 3 patients revealed AMR (an Table 2); 1 ACR, and the other in the same subject (patient 5) chronic rejection with an acute component. One biopsy (patient 9) showed ischemic changes.

Late biliary complications. Patients 1 and 5 presented sclerotic changes in the intrahepatic ducts at cholangiography. Patient 2 had to be retransplanted at 9 months follow-up due to jaundice secondary to severe biliary complications. Patient 9 showed biliary complications at cholangiography before retransplantation.

Death. Five of our patients died after ABOi LT; 3 due to an infection acquired prior to OLT. Patient 3 died at 30 days after transplantation due to multiple septic emboli in the brain; A retrospective analysis of the pretransplantation examinations revealed probable pneumonia as the primary cause, although no agent was isolated. Patient 6 died of

Table 2. Patient Follow-up

\begin{tabular}{|c|c|c|c|c|c|c|c|c|c|c|}
\hline $\begin{array}{l}\text { Patient } \\
\text { No. }\end{array}$ & $\begin{array}{l}\text { Titer } \\
\text { Pre }\end{array}$ & $\begin{array}{l}\text { Titer } \\
\text { MAX }\end{array}$ & $\begin{array}{l}\text { Titer } \\
\text { Pos }\end{array}$ & Pretreatment & Posttreatment & ACR & AMR & $\begin{array}{c}\text { Bilary } \\
\text { Damage }\end{array}$ & $\begin{array}{l}\text { Follow-up } \\
\text { (mo) }\end{array}$ & Patient Status \\
\hline \multirow[t]{2}{*}{1} & 64 & 128 & 1 & No & 8 PP, 1 IvIG, 3 MTP, 2 RTX & - & 1 & Yes & 39 & Alive \\
\hline & 64 & 2048 & 4 & & & & & & & \\
\hline \multirow[t]{2}{*}{2} & 16 & 128 & 16 & Yes PP, IVlg & 3 MTP, 14 P P 11 IVlg 4 RTX & - & 1 & Yes & 9 & Alive $^{*}$ \\
\hline & 128 & 256 & 8 & & & & & & & \\
\hline \multirow[t]{2}{*}{3} & 32 & 256 & 64 & No & $10 \mathrm{IVIg}$ & - & - & - & 1 & Decrease \\
\hline & 32 & 512 & 4 & & & & & & & \\
\hline \multirow[t]{3}{*}{4} & 64 & 64 & 32 & No & 5 PP, 5 IVIG 1RTX & - & - & - & 26 & Alive \\
\hline & 64 & 256 & 25 & & & & & & & \\
\hline & & & 6 & & & & & & & \\
\hline \multirow[t]{2}{*}{5} & 64 & 32 & 1 & Yes PP/RT & 3 PP, 1 RTX, 3 MTP & 1 & - & Yes & 17 & Deceases \\
\hline & - & - & - & $\mathrm{x}$ & & & & & & \\
\hline \multirow[t]{2}{*}{6} & 16 & 64 & 32 & No & $1 \mathrm{RTX}, 1 \mathrm{IVIG}$ & - & - & - & 11 days & Deceases \\
\hline & - & - & - & & & & & & & \\
\hline \multirow[t]{2}{*}{7} & 64 & 256 & 4 & Yes & 5PP, 1 IVIg, 1 RTX & - & - & - & 11 & Alive \\
\hline & 128 & 512 & - & $\mathrm{PP}$ & & & & & & \\
\hline \multirow[t]{2}{*}{8} & 64 & 128 & 1 & Yes & - & - & - & - & 2 days & Decease \\
\hline & 64 & 2048 & 4 & PP & & & & & & \\
\hline \multirow[t]{2}{*}{9} & 32 & 128 & 16 & Yes & - & - & - & Yes & 14 days & Decease $^{\star}$ \\
\hline & - & - & - & RTX & & & & & & \\
\hline \multirow[t]{2}{*}{10} & 16 & 126 & 16 & No & 2 RTX, 6 PP, 4 IVAS & - & 1 & - & 5 & Alive \\
\hline & 16 & 16 & 1 & & & & & & & \\
\hline
\end{tabular}

${ }^{*}$ Retransplanted.

Abbreviations: ACR, acute cellular rejection; AMR, antibody mediated rejection; PP, plasmapheresis; IVIg intravenous immunoglobulin; MTP, methylprednisolone; RTX, rituximab. 
septic MOF on the 11th day after transplantation, suffering recurrent severe systemic infections. Concurrent to OLT ongoing bronchoalveolar lavage and blood cultures showed Pheumocystis Jiroveci plus methicillin-resistant Staphylococcus aureus isolation. Patient 5 died due to septic MOF at 17 months follow-up probably secondary to chronic immunosuppression. Patient 8 died on the 2 nd day after transplantation. He had undergone a first transplantation 2 months prior with several complications thereafter. He presented with severe ischemia of the first graft and was in multiorgan shock at the time of the second transplantation.

Patient 9 was retransplanted with an ABO compatible graft at 14 days due to severely ischemic liver eventually succumbing on the 10th day after the second transplantation due to an hepatic artery thrombosis.

Other infections. Patient 1 presented a cytomegalovirus (CMV) infection in the immediate posttransplantation period. Patient 7 had Staphylococcus Epidermidis isolated in blood cultures and Escherichia coli and Enterococcus Faecalis in the urine. He also presented CMV infection. Patient 9 presented a Pneumocystis Jiroveci infection.

\section{DISCUSSION}

We have described 10 adult emergent ABOi LT patients who displayed $50 \%$ survival despite their severe critical illnesses. It is known that an emergency procedure itself carries higher risk of complications and worse long-term outcomes compared with liver transplantations in nonurgent candidates related to the degree of illness. Published 1-year survival rates have ranged widely from $58 \%$ to $92 \% .^{25}$

In a study of 234 liver transplants, Guggenheim reported 2-year graft survivals for ABO compatible elective (80\%) versus emergency $(76 \%)$ liver transplantation to be significantly higher than for ABOi emergency liver transplantations $(30 \%)$. The poor survival of ABOi LT is therefore related to blood group incompatibility and immunologic damage. ${ }^{7}$ Although ABOi grafts are associated with poor graft survival, long-term patient survival is still good at the cost of a high rate of retransplantations. ${ }^{8-10}$ Recently, Toso et al. did not observe a significant difference regarding patient and graft survival when comparing 1- and 5-year data from $\mathrm{ABO}$, $\mathrm{ABO}$ compatible and $\mathrm{ABO}$ identical livers. ${ }^{11}$

The main complication of ABOi LT is AMR, closely related to high levels of anti-donor $\mathrm{ABO}$ antibodies present perioperative and posttransplant. As a result, most efforts to improve ABOi LT outcome have been directed to reducing anti-blood type antibodies. ${ }^{14,26}$ As a result, most efforts to improve the outcome of ABOi LT have been directed toward lowering anti-blood type antibodies.

We registered four AMR episodes in 3 patients which were all reversible with immunosuppression. This observation is consistent with the literature, as immunological damage is potentially reversible when AMR is mild. It becomes irreversible when there is massive hepatocyte necrosis secondary to vascular thrombosis. ${ }^{12,14}$ These observations reinforce the need for close monitoring of isoagglutinin levels, especially during the first month and argue for prophylactic institution of immunosuppression when levels increase. ${ }^{4,11,13,28}$

We cannot confirm that AMR was directly associated with a higher titer of anti-ABO antibodies. Patients 4 and 5 with elevated titers showed no evidence of AMR or other complications, possibly as a consequence of effective interventions. In addition, patient 9 with relatively low levels experienced biliary complications. The poor positive predictive value of elevated posttransplantation titers has been already reported in otherwise stable ABOi recipients. ${ }^{26}$

There was progressive normalization of the titers after the first month. In fact, 1 month after OLT, most patients presented anti-ABO titers lower than those pretransplantation, whereas others were progressively decreasing. We suppose that the risk of graft dysfunction is mainly present during the first month, tending to vanish later. This is probably related to partial replacement of graft vasculature by endothelial cells that no longer carry foreign $\mathrm{ABO}$ antigens and or to a process of immunological accommodation. $^{21,27}$

In our experience, blood type $\mathrm{O}$ recipients seemed to be associated with a higher risk of AMR after ABOi LT because rejection episodes happened among these recipients. Blood type $\mathrm{O}$ is described in the literature as a significant risk factor for AMR in adults. Group $\mathrm{O}$ patients not only produce immunoglobulin ( $\mathrm{Ig}) \mathrm{M}$ anti-A anti-B, but also a greater degree of T-helper cells, and greater switching to a broader array of IgG subclasses. ${ }^{28}$

In addition to AMR, hepatic artery and biliary complications have been recognized to be the major causes of poor outcomes among these patients. The increased incidence of biliary complications observed in the ABOi group may be related to direct immunological mechanisms, as bile ducts epithelium can also express $\mathrm{ABO}$ antigens, being a target for antibody-mediated injury. ${ }^{15-17}$

A standard therapy was maintained with tacrolimus, mycophenolate mofetil, and prednisolone following basiliximab induction. Pre- and postoperative plasmapheresis targeted isoagglutinin titers of at most 1:16, with or without IVIG and rituximab which were used to deplete cells producing IgM antibodies and those mediating HLA antibody-drives rejection episodes. ${ }^{13,24,29}$

Although splenectomy has been used in desensitization protocols for ABOi transplantation, ${ }^{21}$ it seems that AMR can be avoided with scheduled rituximab prophylaxis or multidrug immunosuppression. ${ }^{14,23}$ Splenectomy avoidance decreases the risk of portal vein thrombosis ${ }^{19}$ and long-term lethal infections. ${ }^{6,23} \mathrm{We}$ also have avoided intrahepatic infusions (portal or arterial) proposed by some authors to control local and disseminated intravascular coagulation. 5,6

Although the recent development of immunosuppression has decreased AMR, the incidence of bacterial infections has increased becoming the most significant cause of death after ABOi LT. ${ }^{5,14,2}$ In fact, one of our major complications 
was infection with the major consequence of MOF resulting in the deaths of 3 patients.

A2 to 0 transplants should be favored when possible, as the antigenic expression of the A2 phenotype is quantitatively and qualitatively different than the A1 phenotype, inducing a weaker immune response. ${ }^{30-32}$

International series have reported that recipient age seems to be a key factor in the outcomes of ABOi LT. We already know that recipients age is significantly related to AMR and that pediatric ABOi LT do not show worse graft survivals versus age-matched OLT. ${ }^{3,5,33}$

These two circumstances, age and donor A2, may permit successful liver transplantations with similar overall and graft survivals even without desensitization. ${ }^{30}$ This opens possibilities to further meet the demands, given the current organ shortage, especially with regard to expanding livingdonor options.

Three of our ABOi LTs were retransplantations. 2 died and the other needed a new transplantation. The specific related circumstances leading to worse outcomes in this situation, have also been reported in other series, ${ }^{10,11}$ suggesting that the use of ABOi grafts for retransplantation may present an even greater challenge, and should be considered only with caution.

In conclusion, the use of ABOi liver grafts can be life saving. They should be offered to all patients in emergent need when no other donors are available. Such transplantations may help to save the patient even at the cost of retransplantation. Our findings support that with careful recipient selection, based on expanding experience and modern immunosuppressive protocols, acceptable outcomes can be obtained. We advocate further expansion to improve the efficacy and safety of ABOi-LT via multicenter trials.

\section{REFERENCES}

1. Starzl TE, lshikawa M, Putnam CW, et al. Progress and deterrents to orthotopic liver transplantation, with special reference to survival, resistance to hyperacute rejection, and biliary duct reconstruction. Transplant Proc. 1974;6:129-139.

2. Todo S, Furukawa H, Jin MB, et al. Living donor liver transplantation in adults:outcome in Japan. Liver Transplant. 2000; 6:66.

3. Stewart ZA, Locke JE, Montgomery RA, et al. ABOincompatible deceased donor liver transplantation in the United States: a national registry analysis. Liver Transplant. 2009;15:883.

4. Tyden G, Donauer J, Wadström J, et al. Implementation of protocol for $\mathrm{ABO}$-incompatible kidney transplantation-a threecenter experience with 60 consecutive transplantations. Transplantation. 2007;83:1153-1155.

5. Egawa H, Teramukai S, Haga $\mathrm{H}$, et al. Present status of ABO-incompatible living donor liver transplantation in Japan. Hepatol. 2008;47:143-152.

6. Kozaki K, Egawa H, Kasahara M, et al. Therapeutic strategy and the role of apheresis therapy for $\mathrm{ABO}$ incompatible living donor liver transplantation. Ther Apheresis Dia. 2005;9:285-291.

7. Gugenheim J, Samuel D, Reynes M, Bismuth H. Liver transplantation across ABO blood group barriers. Lancet. 1990; 336:519-523.

8. Farges O, Kalil AN, Samuel D, et al. The use of ABOincompatible grafts in liver transplantations: a life-saving proce- dure in highly selected patients. Transplantation. 1995;59: $1124-1133$.

9. Chan G, Taqi A, Marotta P, et al. Long-term outcomes of emergency liver transplantation for acute liver failure. Liver Transplant. 2009;15:1696-1702.

10. Bjøro K, Ericzon BG, Kirkegaard P, et al. Highly urgent liver transplantation: possible impact of donor-recipient $\mathrm{ABO}$ matching on the outcome after transplantation. Transplantation. 2003;75: 347-353.

11. Toso C, Al-Qahtani M, Faisal A, et al. ABO-incompatible liver transplantation for critically ill adult patients. Eur Soc Organ Transplant. 2007;20:675-681.

12. Demetris AJ, Jaffe R, Tzakis A, et al. Antibody-mediated rejection of human orthotopic liver allografts. A study of liver transplantation across $\mathrm{ABO}$ blood group barriers. Am J Pathol. 1988;132:489-502.

13. Morioka D, Togo S, Kumamoto T, et al. Six consecutive cases of successful adult ABO-incompatible living donor liver transplantation: a proposal for grading the Severity of antibodymediated rejection. Transplantation. 2008;85:171-178.

14. Egawa H, Ohdan H, Haga $\mathrm{H}$, et al. Current status of liver transplantation across ABO blood-type barrier. J HBP Surg. 2008; 15:131-138.

15. Hayashi S, Noguchi K, Yagihashi A, et al. Expression of blood group antigen (A,B,H, Le(a), Le(b)) on liver allografts. Transplant. Proc. 1992;24:2567-2568.

16. Sanchez-Urdazpal L, Batts KP, Gores GJ, et al. Increased bile duct complications in liver transplantation across the $\mathrm{ABO}$ barrier. Ann Surg. 1993;218:152-158.

17. Nishida S, Nakamura N, Kadono J, et al. Intrahepatic biliary strictures after liver transplantation. J Hepatobil Pancreatic Surg. 2006;13:511-516.

18. Busquets J, Castellote J, Torras J, et al. Liver transplantation across $\mathrm{Rh}$ blood group barriers increases the risk of biliary complications. J Gastrointest Surg. 2007;11:458-463.

19. Testa G, Vidanovic V, Benedetti E, et al. Adult living-donor liver transplantation with ABO-incompatible grafts. Clin Tranaplant. 2008;85:681-686.

20. Urbani L, Mazzoni A, Bianco I, et al. The role of immunomodulation in ABO-Incompatible adult, liver transplant recipients. J Clin Apheresis. 2008;6:55-62.

21. Hanto DW, Fecteau AH, Alonso $\mathrm{MH}$, et al. ABOincompatible liver transplantation with no immunological graft losses using total plasma exchange, splenectomy, and quadruple immunosuppression: evidence for accommodation. Liver Transplant. 2003;9:22-30.

22. Faouzi S, Ichai P, Azoulay D, Samuel D, et al. Successful, long-term, outcome of $\mathrm{ABO}$-incompatible liver transplantation using antigen-specific immunoadorption columns. Ther Apheresis Dial. 2010;14:116-123.

23. Troisi R, Noens L, Montalti R, et al. ABO-mismatch adult living donor liver transplantation using antigen-specific immunoadsorption and quadruple immunosuppression without splenectomy. Liver Transplant. 2006;12:1412-1417.

24. Egawa H, Ohmori K, Haga H, et al. B-cell surface marker analysis for improvement of rituximab prohylaxis in $\mathrm{ABO}$ - incompatible adult living donor liver transplantation. Liver Transplant. 2007;13: $579-588$.

25. Farmer DG, Anselmo DM, Ghobrial RM, et al. Analysing liver transplantation for fulminant hepatic failure: a retrospective look of 18 years' experience. Liver Transplant. 2004;7:666-676.

26. Tobian AA, Shirey RS, Montgomery RA, et al. ABO antibody titer and risk of antibody-mediated rejection in ABOincompatible renal transplantation. Am J Transpl. 2010;10:12471253.

27. Park WD, Grande JP, Ninova D, et al. Accommodation in ABO-incompatible kidney allografts, a novel mechanism of selfprotection against antibody-mediated injury. Am J Transplant. 2003;3:952-960. 
28. Toki D, Ishida $\mathrm{H}$, Horita $\mathrm{S}$, et al. Blood group $\mathrm{O}$ recipients associated with early graft deterioration in living $\mathrm{ABO}$-incompatible kidney transplantation. Transplantation. 2009;88:1186-1193.

29. Kazatchkine MD, Kaveri SV. Immunomodulation of autoimmune and inflammatory diseases with intravenousimmune globulin. N Engl J Med. 2001;345:747-775.

30. Kluger MD, Guarrera JV, Olsen SK, et al; Safety of blood group A2-to-O liver transplantation: an analysis of the United Network of Organ Sharing Database. Transplantation. 2012;94:1-6.
31. Skogsberg U, Breimer ME, Friman S, et al. Successful ABO-incompatible liver transplantation using A2 donors. Transplant. Proc. 2006;38:2667-2670.

32. Rydberg L. ABO-incompatiblity in solid organ transplantation. Transfusion Med. 2001;11:352342.

33. Heffron T, Welch D, Pillen T, et al. Successful ABOincompatible pediatric liver transplantation utilizing standard immunosuppression with selective postoperative plasmapheresis. Liver Transplant. 2006;12:972-978. 